\title{
SALUD MENTAL Y BIOÉTICA: RELACIÓN SIMBIÓTICA
}

\author{
Ernesto A. Frontera Roura*
}

\begin{abstract}
Resumen: El propósito de este trabajo es reflexionar sobre la relación estrecha, de nutrición mutua si se quiere, entre los asuntos relacionados con la salud y las enfermedades mentales, la psiquiatría, la bioética y la salud pública. Se intenta describir y explicar el alcance de cada concepto; incorporar datos epidemiológicos que permitan apreciar la magnitud del problema de salud mental alrededor del planeta; justificar la necesidad de atender esta situación con la organización y operación de un sistema de servicios de cuidado de salud mental que responda a ciertos parámetros éticos, e identificar algunas áreas en las cuales la bioética es un elemento indispensable en el análisis del problema y en la fundamentacion de la solución.
\end{abstract}

Palabras clave: salud mental, enfermedad mental, psiquiatría, bioética, salud pública

\section{MENTAL HEALTH AND BIOETHICS: A SYMBIOTIC RELATIONSHIP}

\begin{abstract}
The purpose of this paper is to reflect about the strong relationship, of mutual nurturance if you will, seen about issues related to mental health and illness, psychiatry, bioethics and public health. An attempt is made to describe and explain the scope of each concept; to incorporate epidemiological data that facilitate us to appreciate the magnitude of the problem of mental health around the planet; to justify the need to attend the situation with the organization and operation of a mental healthcare system of services that respond to certain ethical parameters; and identify some areas in which bioethics is an essential element in the analysis of the problem and in establishing the basis of the solution.
\end{abstract}

Key words: mental health, mental illness, psychiatry, bioethics, public health

\section{SAÚDE MENTAL E BIOÉTICA: RELAÇÃO SIMBIÓTICA}

Resumo: O propósito deste trabalho é refletir a estreita relação, simbiótica, se assim for entendido, entre os assuntos relacionados com a saúde e as doenças mentais, a psiquiatria, a bioética e a saúde pública. Propóe-se descrever e explicar o alcance de cada conceito; incorporar dados epidemiológicos que permitam apreciar a magnitude do problema da saúde mental ao redor do planeta; justificar a necessidade de atender esta situação com a organização e a operacionalização de um sistema de serviços de cuidado à saúde mental que responda a certos parâmetros éticos e identificar algumas áreas nas quais a bioética é um elemento indispensável para a análise do problema e a fundamentação da solução.

Palavras-chave: saúde mental, doença mental, psiquiatria, bioética, saúde pública

Médico Psiquiatra. Puerto Rico

Correspondencia: frontera@caribe.net 
Sanación mental:

No todas las heridas son externas, o sólo debajo de la superficie,

Ocupando un espacio, coagulándose, pustulosas, rojas, enfurecidas.

Fácilmente visibles, palpables, resecables.

Algunas veces las heridas son más profundas;

Dificilmente accesibles, sin una forma física, pero

madurándose;

Silenciosa, incapacitante sin avisar, letal.

Para estas heridas, escuchar atentamente, cuidar

incondicionalmente,

y tranquilizar son vendajes.

Entendimiento es esperanzal.

Venkata Jonnalagadda(1)

Salud mental. Salud de la mente. La mente es el ente del ser humano cuyo sustrato biológico es el sistema nervioso central y periférico y que se relaciona con nuestra capacidad de percibir los estímulos externos e internos, de interpretar esos estímulos y darle significado, de experimentar emociones y sentimientos, y de reaccionar a esos estímulos, significados y emociones mediante la conducta expresada, que nos relaciona con otros seres y organizaciones humanas, con el planeta, el universo y la trascendencia.

Tres factores muy importantes influyen en el desarrollo y la calidad de la mente: 1) el genoma y la biología del cuerpo humano; 2) lo aprendido mediante la experiencia de crecer y vivir en una familia y sociedad compuesta por humanos, animales, plantas y cosas, y 3) las desviaciones patológicas de la mente humana que podríamos llamar "enfermedades mentales".

La primera es el marco de capacidad heredada que impone los límites respecto del grado de crecimiento, desarrollo y cambio, el cual depende de nuestra voluntad. La segunda es el nutrimento experiencial que pone a prueba, reta, enriquece o dańa lo heredado,

1 Mental Healing:

Not all wounds are on the outside, or just beneath the surface.

Occupying a space, clotting, pustulent, red, angry.

Easily visualizad, palpable, resectable.

Sometimes wounds lie deeper;

Difficult to convey, without a physical form, yet ripening;

Silent, crippling without warning, lethal.

For these wounds, attentive listening, unconditional regard,

and reassurance are bandages.

Understanding is hope. y que ocurre a lo largo de toda una vida, generando cambios, adaptaciones y regresiones en el componente sicosocial-trascendental (pensamiento/emoción/ conducta/espiritualidad) de la mente. La tercera se refiere a desviaciones de la normalidad producto de las mutaciones, lesiones, traumas, heridas y defectos que conocemos como "enfermedades mentales", lo suficientemente severas como para alterar la sanidad, estabilidad, funcionalidad y equilibrio de la mente humana en forma temporera o permanente.

La mente humana es el ente ejecutivo del ser humano. Es la mente la que da sentido y dirección a nuestras vidas. Es el conglomerado de mentes humanas lo que le da sentido y dirección a nuestra vida colectiva. No hay salud general si no hay salud mental. No hay salud colectiva si no hay salud mental. El crecimiento, desarrollo, productividad y estabilidad de un pueblo dependen de la salud mental de sus ciudadanos y sus líderes. La mente es la cuna de la percepción, la inteligencia, la memoria, los impulsos motivadores y el lenguaje, además de nuestros pensamientos, ideas, creatividad, sentimientos, decisiones y acciones. En ella también coexisten nuestros instintos e impulsos más egoístas, territoriales, viciosos, agresivos y destructivos, con aquellos altruistas, virtuosos, constructivos y sublimes.

La salud mental es, en parte, responsabilidad personal. Comienza con cuidarse a sí mismo mediante un régimen de vida sano: alimentación balanceada, descanso adecuado, ejercicio moderado, estrés bajo y abstinencia o moderación en el uso de sustancias sicoactivas. Continúa con el cultivo incesante de las virtudes humanas y la supresión también incesante de los vicios, con el objetivo de lograr, a lo largo de la vida, sabiduría, ecuanimidad, estabilidad y felicidad. No todos los elementos que contribuyen a nuestras interpretaciones, decisiones y conductas son conscientes y racionales, de modo que parte de nuestra responsabilidad personal incluye la introspección y reflexión respecto de esos factores inconscientes e irracionales que, en muchas ocasiones, contribuyen a nuestros prejuicios, vicios y conductas inapropiadas. Nutrir nuestro intelecto, cultivar relaciones significativas y enriquecedoras y reflexionar regularmente sobre nuestra vida interna y externa y las consecuencias de nuestra conducta son parte importante de esa agenda de responsabilidad personal. 
Sin embargo, el cultivo y mantenimiento de la salud mental no son exclusivamente un asunto de responsabilidad personal, sino también de responsabilidad social. Vivimos en comunidad, necesitamos de la comunidad para sobrevivir, para proveernos cosas que el ser humano no puede proveerse por sus propios medios. Examinemos brevemente el ciclo de vida del ser humano e identifiquemos los factores sociales que inciden en la salud mental.

Para empezar, el cuidado de la salud mental de un ser humano comienza con el embarazo de su madre: ¿cuán querido es ese embarazo? ¿Cuál es el estado de salud general y mental de sus padres en el momento de la concepción? ¿Cuán sano es el régimen de vida de la madre durante el embarazo? ¿Ha estado expuesta la madre a drogas o sustancias tóxicas? ¿¿Han sido el embarazo y el parto experiencias felices o traumáticas? ¿Han tenido acceso a un sistema de cuidado de la salud adecuado?

Luego del nacimiento y en ausencia de patología, se requiere un cuidado competente, responsable, consistente y amoroso para que el bebé gane confianza en quienes le rodean y cuidan y, como resultado, en sí mismo. Sentirse querido, protegido, seguro y bien cuidado son condiciones esenciales para propiciar un estado de salud mental en el niño/a. La capacidad de los padres para proveer un mínimo básico de satisfacción de las necesidades físicas y emocionales del niño/a es necesaria. ¿Tienen los padres trabajo para cubrir sus necesidades básicas? ¿Tiene acceso el niño/a al proceso de socialización y educación temprana? ¿Es el ambiente alrededor del niño/a uno de uso de drogas, crimen, violencia, pobreza y desempleo?

Más adelante, ¿̇tiene el niño/a acceso a un sistema escolar y de salud adecuados? Como adolescente, ¡̇tiene el espacio para ejercer cierta autonomía practicando para ser adulto, a la vez que la disciplina y el control de adultos que ejemplifiquen una conducta responsable y apropiada? ¿Cuán expuesto está este/a adolescente a las drogas, el maltrato, la violencia y la criminalidad?

Como adulto, ’̨tiene este ser humano la seguridad de un empleo, de una actividad productiva que le permita cubrir sus necesidades y las de su familia, de un sistema educativo que le permita educarse y estimularse intelectualmente, de un sistema de salud general y mental que propicie mantenerse saludable y, en caso de enfermedad, ser atendido, diagnosticado, tratado y recuperado con relativa accesibilidad y prontitud? ¿Tiene las herramientas para cuidarse a sí mismo, su pareja, hijos y padres, trabajar y aportar a la comunidad en que vive? ¿Puede lidiar, cuando le corresponda, con el proceso de envejecimiento, la pérdida de movilidad, funcionalidad, salud y productividad, y enfrentarse al final de su vida con serenidad y sabiduría? ¿Puede confiar en ser bien cuidado hasta el final?

La responsabilidad social aumenta cuando tratamos con seres humanos que, ya al nacer o posteriormente, sufren o padecen una enfermedad mental. Según el DSM-IV-TR “...los trastornos mentales son patrones o síndromes sicológicos o de conducta clínicamente significativos, que ocurren en individuos y que están asociados a un distrés presente (por ejemplo, síntoma doloroso) o discapacidad (por ejemplo, dificultades en una o más áreas de funcionamiento) o con un riesgo significativamente mayor de sufrir muerte, dolor, discapacidad o una importante pérdida de libertad. Además, este síndrome o patrón no debe ser meramente una respuesta esperada o sancionada culturalmente a un evento particular, por ejemplo, la muerte de un ser querido. Cualquiera que sea su causa original, debe considerarse en el presente como una disfunción conductual, sicológica o biológica en el individuo. $\mathrm{Ni}$ una conducta atípica (política, religiosa o sexual) ni conflictos principalmente entre el individuo y la sociedad son trastornos mentales, a menos que lo atípico o el conflicto sean un síntoma de una disfunción en el individuo, según se ha descrito arriba”(2).

Los trastornos mentales clasificados en el DSM-IV-TR pueden, dependiendo de su intensidad, afectar la salud mental de una persona, comprometiendo, en menor o mayor grado, su capacidad para percibir, interpretar, sentir y actuar con buen juicio, equilibrio, prudencia y cordura. En otras palabras, afecta la funcionalidad, productividad y capacidad de la persona para ejercer su autonomía personal, implementar su agenda de vida y formar parte en plenitud de la comunidad de humanos a la que pertenece.

El estado de incomprensión e indefensión que genera la enfermedad mental hace de las personas que la padecen objeto de estigma, prejuicio, maltrato y marginación. Además, genera en sus familias y en la sociedad una demanda de tiempo y recursos materiales para su atención y cuidado, así como su propia pérdida de productividad. 
Veamos algunos datos que nos proveen los estudios epidemiológicos de la Organización Mundial de la Salud (OMS) y publicados en el libro "The WHO World Mental Health Surveys. Global Perspectives on the Epidemiology of Mental Disorders", editado por Ronald C. Kessler \& T. Bedirhan Ustun(3). Nos dan una idea de la magnitud del problema de salud mental y el impacto que tiene en dimensiones como la productividad, el presupuesto personal y gubernamental y otras:

- La prevalencia de por vida de los trastornos mentales analizados en este estudio (trastornos afectivos, de ansiedad, de control de impulsos, y uso de sustancias) es de $18,1 \%$ a $36,1 \%$.

- Las prevalencias por trastornos son las siguientes:

1. Trastornos de ansiedad: $4,8 \%-31,0 \%$

2. Trastornos del estado de ánimo: 3,3\% $21,4 \%$

3. Trastornos de control de impulsos: $0,3 \%$ $25,0 \%$

4. Trastornos de uso de sustancias: $1,3 \%$ $15,0 \%$

- La co-ocurrencia de estos trastornos a lo largo de la vida parece ser bastante común.

- La edad de comienzo para los distintos trastornos es la siguiente:

1. Trastornos de control de impulsos:

a. Trastorno de déficit de atención/hiperactividad: 7-9 años de edad

b. Trastorno oposicional-desafiante: 7-15 años de edad

c. Trastorno de conducta: 9-14 años de edad

d. Trastorno explosivo intermitente: 13-21 años de edad

2. Trastornos de ansiedad:

a. Trastornos de ansiedad de separación y fobias: 7-14 años de edad

b. Trastornos de ansiedad generalizada, pánico y estrés postraumático: 24-50 años de edad

3. Trastornos del estado de ánimo: 29-43 años de edad

4. Trastornos de uso de sustancias: adolescencia y adultez joven

- La razón riesgo-prevalencia más alta se encuentra en países expuestos a violencia sectaria (Israel, Nigeria y África del Sur).
- El riesgo proyectado de por vida de cualquiera de los trastornos a la edad de 75 ańos es de 17\% más alto en Estados Unidos y $69 \%$ más alto en Israel.

- El riesgo proyectado más alto (45\%-170\%) corresponde a los trastornos del estado de ánimo.

- Se calcula que, aproximadamente, entre $18 \%$ y $55 \%$ de la población mundial sufrirá por lo menos un trastorno mental a lo largo de su vida, dependiendo del país donde viva.

- Otros hallazgos:

a. Los jóvenes y los viejos son renuentes a buscar ayuda.

b. Las mujeres buscan ayuda con más frecuencia que los hombres.

c. Los más educados buscan ayuda con más frecuencia.

d. Los más ricos en los países desarrollados y los países en desarrollo son los que más ayuda buscan.

e. Los no casados usan más los servicios de cuidado.

f. Los trastornos mentales ocurren con frecuencia y tienen una sintomatología parecida en diferentes culturas.

g. También es similar la alta frecuencia de trastornos de ansiedad y del estado de ánimo en mujeres, y de trastornos de control de impulso y relacionados con sustancias en hombres.

h. Solo una minoría de los trastornos mentales son severamente incapacitantes.

i. La incapacidad asociada con trastornos mentales es consistentemente más alta, en promedio, que la incapacidad asociada con trastornos físicos, como el asma, el cáncer, la diabetes y las condiciones cardíacas. Aun los trastornos mentales leves son más incapacitantes que muchos trastornos físicos crónicos.

j. Existe un problema de tratamiento de baja calidad en todos los países estudiados para estas condiciones. Un factor de peso en este problema es la falta de seguimiento en el tratamiento.

k. Áreas de estudio futuro incluyen: correlaciones sociodemográficas importantes; factores de riesgo substantivos (adversidades en la niñez, experiencias traumáticas de vida); barreras al tratamiento (patrones y determinantes de baja 
del tratamiento); costos sociales incluyendo el efecto de comienzo temprano en el proyecto de vida (bajo aprovechamiento educativo, matrimonios tempranos, inestabilidad marital, ingresos bajos); efecto de un trastorno mental reciente en el funcionamiento reciente de su rol; comorbilidad entre los trastornos mentales y físicos, y la epidemiología del suicidio (ideación, planes, gestos e intentos).

- En un estudio realizado por la Alianza Europea contra la Depresión, el suicidio es un problema severo de salud pública en la Unión Europea, producto y causa de sufrimiento. En esa región 58.000 personas mueren anualmente como resultado del suicidio; según la OMS, una de las primeras diez causas de muerte en el mundo para todas las edades. Se calcula que el número de intentos no fatales de suicidio es 10 a 20 veces los logrados(4).

Kessler y Ustun llegan, entre otras, a las siguientes conclusiones:

- Estos desórdenes son bastante comunes en todos los países estudiados y, por lo tanto, son muy importantes en el manejo de la salud mental en el mundo.

- Dada la carga enorme personal y social de los trastornos mentales, el hallazgo de que muchos de los casos tienen una edad de comienzo temprana sugiere que las intervenciones de salud pública deben comenzar en la niñez.

- Estudios epidemiológicos sugieren que los siguientes factores están asociados al comienzo temprano de trastornos mentales no tratados: fracaso escolar y de trabajo, preñez en la adolescencia y matrimonios tempranos, violentos o inestables.

- Un trastorno mental frecuentemente evoluciona a trastornos comórbidos complejos más difíciles de tratar y recurrentes. Aún los trastornos leves, si no se atienden o tratan, evolucionan a una mayor severidad, comorbilidad siquiátrica adicional y funcionamiento social y ocupacional negativo.

- Muchos casos de trastornos mentales buscan ayuda años después del comienzo del trastorno, problema común en los países en desarrollo, en los cuales las barreras estructurales y financieras para acceder a servicios parecen ser mucho mayores.
Poco se sabe sobre el impacto que las políticas nacionales, el diseño de sistemas de provisión de servicios y los modos de financiamiento tienen en la provisión de la identificación y cuidado temprano de los trastornos mentales.

- Los estudios de prevalencia en un período de doce meses son consistentes con estudios previos en demostrar la alta prevalencia de los trastornos mentales y su alta correlación con disfunción seria en los roles de las personas que los padecen, especialmente en la medida en que la severidad sea mayor.

- El estudio sobre la carga global de la enfermedad de la OMS ("WHO Global Burden of Disease Study”) concluyó que las condiciones neuropsiquiátricas son las causas principales de incapacidad en el planeta, responsables de la pérdida del 37\% de todos los años de vida saludable.

- Sin embargo, el estudio de la OMS concluye que han encontrado altos niveles de ausencia de necesidades satisfechas en cuanto a tratamiento en salud mental en el mundo, aun entre aquellos que padecen desórdenes más severos. La situación parece ser más grave en los países en desarrollo, en los cuales sólo una fracción pequeña de las personas con trastornos más severos reciben algún tipo de cuidado. Pero, incluso en los países desarrollados, alrededor de la mitad de los casos severos no recibe ayuda. Más aún, entre aquellos pocos que reciben servicios son menos los que reciben tratamiento efectivo.

- A pesar de que cada vez hay más herramientas terapéuticas eficaces y tolerables disponibles, la competencia en prioridades y las limitaciones presupuestarias limitan su utilización. Se hace imperativo entonces determinar cómo proveer un cuidado de la salud y de los trastornos mentales efectivo.

- En muchos países, una cuarta parte de los que inician su tratamiento no recibe seguimiento. Consistente con estudios anteriores, se observó que sólo una minoría de los tratamientos llenaban criterios mínimos de adecuación.

- Según los hallazgos del Proyecto Atlas de la OMS, en casi todos los países los gastos en salud mental son mucho menores que lo necesario, tomando en 
cuenta la magnitud estimada de la carga social de las enfermedades mentales. La mayoría de los casos tratados corresponde a trastornos leves, mientras los casos más severos no son atendidos. Esto plantea la posibilidad de que los pocos recursos existentes no estén siendo bien utilizados.

- Se observó también que en la mayoría de los países estudiados la fuente primaria de servicios de salud mental ocurre en el sector de medicina general. Se necesitan estudios posteriores para asegurar que estos servicios no sean de poca adecuación e intensidad. Por lo tanto, hay que enfocarse en mejorar la calidad de los servicios de salud mental en el entorno de medicina general.

- Según este estudio, para que un país pueda rediseñar su sistema de cuidado de salud mental y distribuir los recursos óptimamente requiere:

a. Documentar los servicios utilizados en el presente así como la naturaleza y magnitud de las necesidades no satisfechas de tratamiento.

b. Identificar la fuente de provisión de servicios: medicina general, especialidades de salud mental, medicina complementaria y alternativa, servicios humanos, entre otros.

c. Dar prioridad al ofrecimiento de servicios a las personas con trastornos más severos, enfatizando la adecuación del tratamiento y las visitas de seguimiento.

d. Revisar no sólo la proporción de los recursos fiscales que se dedican a ofrecer servicios de cuidado en salud mental, sino también las prioridades dentro del sistema de cuidado (ingresos bajos y severidad alta vs. medios y altos ingresos y severidad leve).

e. Establecer prioridades en la distribución de recursos de manera racional, transparente y ética.

El efecto devastador que la enfermedad mental puede generar en un ser humano demanda del apoyo, solidaridad y cuidado de la sociedad, mediante un sistema de atención de la salud mental integrado al sistema de la salud general. A pesar de que existen múltiples modalidades de tratamiento efectivos para los trastornos mentales y de que se ha evidenciado la importancia de la salud mental, son pocos, si alguno, los países que han dado prioridad a este asunto en su agenda gubernamental y presupuestaria. Los datos de estudios preclínicos sugieren que, mientras más temprano se traten los trastornos mentales -o sea, antes de que se desarrolle la secuela negativa-, menos frecuentes, espontáneos, severos y refractarios a tratamientos serán(3). Es interesante notar la poca o ninguna alusión sobre salud mental en el presente debate sobre la reforma del sistema de cuidado de la salud en Estados Unidos, a pesar de las críticas hechas en el President's New Freedom Commission on Mental Health de 2003(5).

En 2003, la Administración de Servicios de Salud Mental y Contra la Adicción (ASSMCA) del Estado Libre Asociado de Puerto Rico nombró un grupo de trabajo, coordinado por mí, para, entre otros temas, identificar las metas y principios generales que deben guiar la organización y operación de un sistema de cuidado de la salud mental de un país, en este caso Puerto Rico(6). Según este grupo, las metas de este sistema de cuidado deben ser:

- La educación sobre un régimen de vida sano, la promoción de su vivencia y la prevención de las enfermedades mentales, de manera que menos personas padezcan de estas condiciones.

- El alivio del dolor y el sufrimiento causados por estas enfermedades, tanto a las personas que los padecen como a sus familiares, de manera que mejore su calidad de vida.

- El cuidado y tratamiento de las personas con trastornos mentales, integrándolos al cuidado de la salud general.

- La recuperación y rehabilitación de las personas que padecen de trastornos mentales, de manera que puedan vivir una vida autónoma y productiva.

- Combatir y, si posible, eliminar el estigma asociado con estas condiciones.

- Investigar las causas y los factores que precipitan, sostienen o agravan estas condiciones, así como las modalidades de tratamiento más efectivas disponibles.

- Evaluar los resultados de sus intervenciones basados en la evidencia científica.

- Asegurarse que, operacional y administrativamente, el sistema de cuidado de salud mental se base en los principios bioéticos fundamentales de respeto 
a la dignidad y autonomía de las personas, beneficencia y no-maleficencia y justicia, garantizando el acceso, la equidad, la efectividad, la eficiencia y la eficacia.

- Preparar académicamente a los profesionales de salud mental que proveerán el cuidado directo a pacientes y familiares.

- Asumir un rol de liderato y coordinación con organizaciones de la comunidad y otras agencias del gobierno, en lo que se refiere a la agenda pública relacionada con la salud, la educación, el desempleo, la pobreza, la drogadicción, la criminalidad, la violencia, el discrimen y la desigualdad, y otros factores socioeconómicos que inciden en la salud mental, ya sea como causas, efectos o facilitadores de los trastornos mentales.

Dicho informe afirmaba también que, para que un sistema de cuidado de salud mental integrado al cuidado de la salud general responda a un imperativo ético de excelencia, debe ser guiado por los siguientes principios generales:

- Prioridad: La salud mental está presente en todas las actividades del ser humano. Si bien la prioridad máxima en Puerto Rico es la lucha contra el desempleo y la pobreza, existe un círculo vicioso de pobreza y trastornos mentales, incluyendo el abuso de sustancias, que hace que su mayor prevalencia y la insuficiencia de los sistemas de prevención, tratamiento, cuidado y rehabilitación tenga repercusiones económicas (pérdida de empleo y reducción de la productividad), que agravan y perpetúan la condición de pobreza de las familias. Por esta razón, en la agenda de gobernar el país, así como en las expresiones públicas y oficiales de nuestros dirigentes políticos y gubernamentales, el cuidado de la salud mental debe ser de la más alta prioridad.

- Responsabilidad gubernamental: El gobierno, como garante del bien común y respondiendo a las necesidades y voluntad del pueblo, es responsable de que exista un sistema de cuidado de salud mental articulado, ágil, lo menos restrictivo posible, fiscalmente responsable, estable y compasivo, independientemente de a quién se deleguen las tareas específicas de su operación, sea el sector público, privado (con o sin fines de lucro) o una combinación de los mismos.
- Paradigma bio-psico-social-espiritual: Las intervenciones en el manejo de los trastornos mentales y de conducta se clasifican en cuatro categorías: prevención, tratamiento, cuidado y rehabilitación. A lo largo de este continuo, el sistema de cuidado de salud mental debe guardar una estrecha relación no sólo con el cuidado médico en general, sino también con las agencias públicas, instituciones privadas y organizaciones no gubernamentales que lidian con los diversos problemas biológicos, psicológicos, sociales, espirituales y económicos de la población.

- Revisión dinámica y continua: La implementación de un sistema de servicios de salud y, más aún, de salud mental, debe llevarse a cabo junto con una revisión dinámica y continua. Desde que el cuidado de la salud mental se inició hasta el presente, no se ha podido articular e implementar una política pública capaz de organizar un sistema que satisfaga las preocupaciones, necesidades y aspiraciones de nuestros pacientes y familiares, los profesionales que los atienden y los intermediarios(6). Desde la implementación hace alrededor de diez años de los cambios en nuestro sistema de salud, y específicamente en el de salud mental, se ha generado un debate público tanto sobre los aspectos filosóficos y de política pública como sobre los operacionales y económicos que parece no terminar. Luego de estos ańos de cambios es necesario volver a la mesa de trabajo, revisar lo que se ha hecho y plantear preguntas sobre lo que debe ser este sistema, sobre su estructura, herramientas operacionales y fuentes de financiamiento.

- Responsabilidad financiera compartida: Existe consenso en cuanto a que el Estado, la empresa privada, las organizaciones comunitarias y los ciudadanos deben compartir los costos del sistema de cuidado de salud mental en forma justa y equitativa. En efecto, la magnitud del costo de cada una de las múltiples dimensiones del sistema de salud mental -entre ellas la educación en salud, el cuidado de pacientes, la investigación y desarrollo de medicamentos, los sistemas de información, la formación de los profesionales de la salud, la inversión en equipo y facilidades físicas, y la creación de redes de apoyo comunitario- obliga a que la responsabilidad financiera sea compartida entre los diferentes sectores de la sociedad e, inclusive, 
conlleva la búsqueda del apoyo de organismos internacionales.

El cuidado de la salud mental debe ser prioridad en la agenda de gobernar un país. Personas con enfermedades mentales severas mueren 25 años más temprano que la población general "debido mayormente a condiciones médicas tratables que son causadas por factores de riesgo modificables como el fumar, la obesidad, el abuso de sustancias y acceso inadecuado a cuidado médico", según los hallazgos de un informe de la National Association of State Mental Health Program Directors de los EUA, en 2006(7).

Kathryn Power(8) sugiere que los principios generales que deben guiar la transformación de los servicios de salud mental en EUA deben dirigirse a constituir un tipo de servicio: centrado en la persona; enfocado en la recuperación; basado en la evidencia; dirigido a la calidad, y orientado a los resultados.

He discutido hasta ahora los conceptos de mente y salud mental, los aspectos biológicos y sicosociales necesarios para propiciar la salud mental en términos del ciclo de vida del ser humano, la definición de enfermedad mental, su prevalencia mundial, el impacto en la población, la necesidad de un sistema de servicios de cuidado de la salud mental y los parámetros a tomar en cuenta en su organización, operación y financiamiento. Pasemos ahora a discutir la relación entre psiquiatría y bioética.

La salud mental y la psiquiatría se nutren de la bioética para contribuir en la realización del proyecto de vida de cada ser humano. Considero que en las siguientes áreas el trabajo conjunto entre la psiquiatría y la bioética es indispensable:

a. En el desarrollo del carácter y las virtudes personales que debemos cultivar a lo largo de la vida y en la identificación de la naturaleza destructiva y corruptora de los vicios humanos. Aun cuando es difícil promover o cambiar rasgos de carácter luego de la adolescencia y la adultez joven, no es del todo imposible y, además, podemos fortalecer las virtudes. Por esta razón, en los programas de educación y preparación de profesionales el ambiente de entrenamiento debe fomentar y nutrir la aplicación de las virtudes al trabajo clínico. El énfasis en la competencia en ética de los profesionales de la salud mental está íntimamente ligada con la calidad en el cuidado del paciente(9). Se trata de propiciar la integridad entre lo que pensamos, sentimos, decimos, juramentamos y actuamos como profesionales de la salud.

b. En la búsqueda de una definición contemporánea de lo que es una vida buena, correcta y apropiada y por qué es importante para el ser humano incorporarla a su proyecto de vida. No sólo hay que entender qué nos mueve sino qué nos debe mover, y cómo alcanzar esa vida buena.

c. En la identificación de asuntos que plantean aspectos morales y cómo, utilizando el proceso de razonamiento moral, nos acercamos a ellos, propiciando la deliberación y el consenso en medio de un contexto pluralista y diverso.

d. En delimitar las fronteras de las relaciones interpersonales (relación profesional/paciente/familia/otros profesionales) que se desarrollan en el contexto clínico. Es un contexto en el cual la solidaridad, la colaboración, el apoyo y la compasión deben reinar por encima de lo adversativo, lo competitivo, la insensibilidad y la explotación.

e. En delimitar las fronteras de las relaciones entre el paciente/familia y el profesional de la salud con instituciones intermediarias como hospitales, aseguradoras, organizaciones de manejo coordinado, industria farmacéutica, organizaciones profesionales, instituciones académicas y el gobierno.

f. En establecer los fundamentos, guías y principios éticos que deben orientar la organización, administración, operación y financiamiento de un sistema de cuidado de la salud (mental y general).

g. En contribuir a la justificación de las prioridades presupuestarias y de servicios basadas en valores, severidad, necesidad, acceso y resultados.

h. En contribuir a distinguir la conducta criminal de la conducta insana. En delimitar la capacidad y responsabilidad de una persona en función de su conducta y las consecuencias de ésta en el campo de la psiquiatría forense.

i. En ayudar a delimitar la frontera y el equilibrio entre la responsabilidad personal y la responsabilidad social.

j. En la formulación de guías y salvaguardas en el uso de sujetos humanos para las investigaciones en salud mental y general, así como en lo relacionado con la conducta de los investigadores.

k. En la elaboración de parámetros éticos relacionados con la evaluación continua de las intervenciones terapéuticas y organizacionales. 
1. En enmarcar los servicios de cuidado de la salud con relación al ambiente y la preservación del planeta para futuras generaciones.

No hay bioética sin salud mental; no hay salud mental sin bioética.
Agradecimientos: Agradezco la orientación y guía que Jorge J. Ferrer Negrón me ha provisto por ańos en mi proceso autodidacta de profundizar en el estudio de la bioética e incorporarla a mi proceso personal de analizar situaciones y problemas y tomar decisiones tanto en lo personal como en lo profesional. Su estímulo y apoyo fueron determinantes en aceptar el reto de escribir este trabajo.

\section{Referencias}

1. Jonnalagadda V. Mental Healing. Brief Report. Academic Psychiatry 2009; 33:156.

2. American Psychiatric Association. Diagnostic and Statistical Manual of Mental Disorders, Fourth Edition, Text Revision. DSM-IV-TR. Arlington, VA.: APPI; 2000.

3. Kessler RC. (Harvard Medical School), Ustun TB (World Health Organization) (eds.) The WHO World Mental Health Surveys. Global Perspectives on the Epidemiology of Mental Disorders. Published in collaboration with the World Health Organization, Geneva, Switzerland. New York; Cambridge University Press; 2008.

4. Hegerl U, Wittenburg L. Focus on Mental Health Care Reforms in Europe: The European Alliance Against Depression: A Multilevel Approach to the Prevention of Suicidal Behavior. Column. Psychiatric Services 2009; 60: 596-599.

5. Department of Health and Human Services. Achieving the Promise: Transforming Mental Health Care in America. Pub no SMA-03-3832. Rockville, Md: President's New Freedom Commission on Mental Health; 2003.

6. Administración de Servicios de Salud Mental y Contra la Adicción (ASSMCA). Estado Libre Asociado de Puerto Rico. Informe del Grupo de Trabajo sobre Politica Pública en relación a Sistema de Cuidado de Salud Mental; 2003.

7. Schuffman D, Druss BG, Parks, JJ. State Mental Health Policy: Mending Missouri’s Safety Net: Transforming Systems of Care by Integrating Primary and Behavioral Health Care. Column. Psychiatric Services 2009; 60: 585-588.

8. Power AK. Focus on Transformation: A Public Health Model of Mental Health for the 21st Century. Column. Psychiatric Services 2009; 60: 580-584.

9. Block, S, Green SA. Promoting the Teaching of Psychiatric Ethics. Commentary. Academic Psychiatry 2009; 33: 89-92.

Recibido: 14 de agosto de 2009

Aceptado: 25 de agosto de 2009 